\title{
The Effect of Chitooligosaccharides on Gut Microbiota in Diabetic Mice
}

\author{
Xin Wang*, Qin Hu, Chao Dong \\ School of Medicine, Ningbo University, Ningbo, China \\ Email: *wangxin@nbu.edu.cn
}

How to cite this paper: Wang, X., Hu, Q. and Dong, C. (2019) The Effect of Chitooligosaccharides on Gut Microbiota in Diabetic Mice. Open Access Library Journal, 6: e5961. https://doi.org/10.4236/oalib.1105961

Received: November 27, 2019

Accepted: December 21, 2019

Published: December 24, 2019

Copyright $\odot 2019$ by author(s) and Open Access Library Inc.

This work is licensed under the Creative Commons Attribution International License (CC BY 4.0).

http://creativecommons.org/licenses/by/4.0/

\begin{abstract}
Chitooligosaccharides (COS) has an effect to reduce blood glucose and blood lipids and is used in adjuvant therapy for diabetes mellitus (DM), which is closely associated with gut microbiota. Therefore, it is worthy investigating whether COS exerts its effect via the gut microbiota. In this paper, we studied the response of gut microbiota in diabetic mice to COS, using the high-throughput sequencing technology. We found that COS had significant effect on the community structure of gut microbiota in mice though it had no significant effect on the $\alpha$-diversity of gut microbiota; the abundance of Actinobacteria was increased significantly in the gut tract of mice fed with COS $(P<0.05)$; the abundance of Corynebacteriaceae significantly increased in diabetic mice fed with $\operatorname{COS}(P<0.05)$; at OTU level, the abundance of 13 OTUs of Bacteroidales S24-7 group decreased significantly $(P<0.05)$ while that of Lachnospiraceae increased significantly $(P<0.05)$ in diabetic mice intragastrically administered with COS. The results of our experiment suggest that the effect of COS to reduce blood glucose in diabetic mice might be produced by decreasing the abundance of Bacteroidetes and increasing that of Firmicutes and Actinobacteria.
\end{abstract}

\section{Subject Areas}

Diabetes \& Endocrinology

\section{Keywords}

Diabetes, Chitooligosaccharides, High-Throughput Sequencing, Gut Microbiota

\section{Introduction}

Recently, more and more studies have shown that gut microbiota, as one of the various environmental factors, is closely associated with a number of diseases 
such as obesity and DM [1] [2] [3] [4]. High-throughput sequencing of 16SrRNA gene of gut microbiota in healthy human revealed that gut microbiota is mainly composed of Bacteroidetes, Firmicutes, Actinobacteria, Fusobacteria, Verrucomicrobia, Cyanobacteria, Proteobacteria, Spirochaeates and VadinBE97 [5], of which Bacteroidetes, Firmicutes, Actinobacteria and Proteobacteria account for the most part of gut microbiota; in particular, Bacteroidetes and Firmicutes dominate and account for more than $95 \%$ of the gut microbiota [6]. The community structure of gut microbiota differs significantly between diabetic patients and healthy human. In patients with type II DM, the percentage of class Clostridia bacteria in phylum Firmicutes in gut microbiota decreases significantly; the ratio of Bacteroidetes to Firmicutes is positively correlated with the concentration of blood glucose; and the abundance of bacteria that produce butyric acid decreases; various pathogenic bacteria increase; and there is a phenomenon of significant enrichment of Betaproteobacteria, which increases with the increase of blood glucose concentration [7].

Chitin has the effect of reducing blood pressure, blood lipids and blood glucose, regulating immunity and suppressing tumor and is suitable for adjuvant treatment of DM, hypertension, hyperlipidemia, obesity and tumor. It is widely used in studies on reduction of blood glucose and blood lipids in mice [8] [9] [10]. Recently, some studies reported that chitin has the function to modulate gut microbiota, for example, to significantly reduce the quantity of Bifidobacteria in gut microbiota in diabetic mice [11]. Nonetheless, these studies only tested change in the quantity of a few key gut bacteria using traditional culture technology or at most investigated microbiota change using DNA fingerprinting technology [12] [13] [14] and thus very limited information was revealed. Using $16 \mathrm{~S}$ rRNA gene high-throughput sequencing technology for assaying gut microbiota in mice to obtain a large gene sequence may greatly deepen the understanding of the mechanism of COS modulating gut microbiota and mice blood glucose.

In this study, we employed the $16 \mathrm{~S}$ rRNA gene high-throughput sequencing technology on diabetic mice model fed with COS to investigate: 1) whether or not COS would cause change in the composition structure of gut microbiota in diabetic mice, and 2) if such change exists, whether or not it is associated with change in blood glucose. Results of the study will provide a theoretical basis for the use of COS as a mean to improve prognosis of DM and modulate blood glucose of diabetic patients as an adjuvant of medicine control.

\section{Materials and Methods}

\subsection{Experimental Animals}

Animals used in this study were SPF grade male ICR mice weighted $20 \pm 2 \mathrm{~g}$, purchased from the Comparative Medicine Center of Yangzhou University (Yangzhou, Jiangsu, China) [Permit Number: SCXK (S)2017-007]. The animal feed was provided by Zhejiang Experimental Animal Center (Zhejiang, China). 
All experiment protocol were accordance with the standard animal welfare guideline, and obtained the permission should be obtained from Ethics Committee.

\subsection{Reagents and Instruments}

Streptozocin-STZ was purchased from Sigma Corporation (USA). Citric acid and sodium citrate were purchased from Shanghai Kejian Biotechnology Co., Ltd. (Shanghai, China). COS was obtained from Ningbo Zhenhai Baichuan Biotechnology Co., Ltd. (Ningbo, Zhejiang, China).

Cofoe blood glucose meter and Cofoe blood glucose test paper were provided by Cofoe Medical Equipment Co., Ltd. (Changsha, China). QIAamp DNA Stool Mini Kit was obtained from Qiage Corporation (Germany). NanoDrop ND-2000 Nucleic acid analyzer and GeneJET Gel Extraction Kit were obtained from Thermo Scientific (USA). Illumina HiSeq 2500 Sequencing Platform was from Illumina (USA).

\subsection{Animals Grouping and Treatment}

After one week of acclimatization, 30 out of 50 mice were randomly selected and divided into the experiment group for DM modeling and were intraperitoneally injected with Streptozocin-STZ diluted with citric acid and sodium citrate lactated ringer's solution in a dose of $200 \mathrm{mg} / \mathrm{kg}$ after fasting for $12 \mathrm{~h}$ [11]. The remaining 20 mice were divided into the normal blood glucose group and intraperitoneally injected with an equivalent amount of purified water. Three days after the injection, blood samples were collected from the tail vein of mice in both groups and levels of blood glucose were determined with the Glucose Oxidase method. Mice in the experiment group with blood glucose level exceeding 11 $\mathrm{mmol} / \mathrm{L}$ were deemed as successful DM modeling and used for further experiment. The blood glucose levels of the normal group were between 3.5 and 7.1 $\mathrm{mmol} / \mathrm{L}$ [15].

Mice in the DM model group were divided into two groups according to the principle of similar weights: DM mice group intragastrically administered with COS (the DM-T group) and DM mice control group (DM-C). Similarly, mice in the normal glucose group were also divided into group intragastrically administered with $\mathrm{COS}$ (the $\mathrm{N}-\mathrm{T}$ group) and control group (N-C). Each group had six mice. Mice in the DM-T group and N-T group were intragastrically administered with COS $(600 \mathrm{mg} / \mathrm{kg} / \mathrm{d})$ [16], while those in the N-T and N-C were intragastrically administered with an equivalent amount of purified water for 4 consecutive weeks. Each week all mice in both experiment groups and control groups were monitored for level of blood glucose and weight. At W4, mice feces were collected under aseptic conditions. During the experiment, mice in each group were housed in one cage under a $12 \mathrm{~h}$ light- dark circle with controlled humidity $(40 \%-70 \%)$ and temperature $\left(20^{\circ} \mathrm{C}-26^{\circ} \mathrm{C}\right)$ and were given unrestricted access to water and feed. All utensil, feed and bedding were sterilized. 


\subsection{Collection, DNA Extraction, PCR Amplification and Purification of Mice Feces}

At the end of treatment, mice were sacrificed. After the low abdomen external skin of mice was sterilized with $75 \%$ alcohol, feces in the rectum end were collected in sterile cryogenic tubes, marked and immediately stored in liquid nitrogen. The total microbial DNA of mice feces was extracted using QIAamp ${ }^{\infty}$ DNA Stool Mini Kit (Qiagen, Germany). The concentration and purity of DNA was measured using NanoDrop ND-2000 Nucleic acid analyzer. The DNA samples were stored at $-80^{\circ} \mathrm{C}$. The V4-V5 region of $16 \mathrm{~S}$ rRNA gene was amplified using ancient and bacterial universal primers 515FB (5'-GTGYCAGCMGCCGCGGTAA-3') and 926R (5'-CCGYCAATTYMTTTRAGTTT-3') [17]. To minimize reaction-level PCR bias, $10 \mathrm{ng}$ purified DNA template from each sample was amplified in triplicate with a $30-\mu \mathrm{L}$ reaction system under the following conditions: initial denaturation at $95^{\circ} \mathrm{C}$ for $3 \mathrm{~min}$; then 25 cycles of denaturation at $95^{\circ} \mathrm{C}$ for $45 \mathrm{~s}$, annealing at $50^{\circ} \mathrm{C}$ for $45 \mathrm{~s}$ and extension at $68^{\circ} \mathrm{C}$ for $90 \mathrm{~s}$, with a final extension at $68^{\circ} \mathrm{C}$ for 5 $\mathrm{min}$. At the end of amplification, products of the same sample were mixed with equal concentration and detected by $2 \%$ agarose gel electrophoresis. Sequences between $400 \mathrm{bp}$ and $450 \mathrm{bp}$ were selected and target bands were excised using GeneJET Extraction Kit (Thermo Scientific, USA). After purification of the PCR product, the library was constructed using the TruSeq DNA PCR-Free Library Preparation Kit (Illumina, USA) and sequenced on the Illumina HiSeq 2500 platform (Illumina, USA).

\subsection{Analysis of Sequencing Data}

The raw FASTQ files were packaged using QIIME v.1.9.1 [18] and the paired reads were spliced with FLASH [19]. The joined pairs were subsequently quality filtered and analysed with QIIME v1.9.1. The sequences were clustered into operational taxonomic units (OTUs) at $97 \%$ similarity using the pick_open_reference_otus.py script. The most abundant sequence from each OTU was selected as a representative sequence, and compared with SILVA128 database and assigned to taxonomy. A phylogenic tree was generated from the filtered alignment using FastTree [20]. Sequences identified as archaea, chloroplast, mitochondria and not classified into bacterial were removed. A total number of 1,206,800 sequences were obtained for 24 samples (with a mean of 50,283 reads per sample). Each sample was then standardized to the minimum number of 43,100 sequences (the lowest number of sequences in all samples) for further analysis.

The a-diversity indices (observed species, Shannon-Wiener index and phylogenetic diversity) and $\beta$-diversity (Bray-Curtis dissimilarity) were calculated using QIIME v1.9.1. Pielou's evenness of mice fecal microbiota was calculated using the "Vegan" package in R. One-way analysis of variables (ANOVA) was used to test the differences of bacterial a-diversity among different mice fecal groups, respectively, with SPSS 22.0. Principal coordinate analysis (PCoA) based on Bray-Curtis dissimilarity was used to indicate the difference in the community 
structure of mice fecal microbiota across the four treatment groups. A permutational multivariate analysis of variance (PERMANOVA, with the "adonis" function in the "vegan" package) was applied to assess the effect of COS treatment on the community structure of mice fecal microbiota.

\section{Experiment Results}

\subsection{Change in Weight and Blood Glucose of Mice}

The changes in body weights of mice in each group over time are shown in Figure 1(A). The body weights of mice in N-C, N-T and DM-T increased over time and at each time point presented the order of $\mathrm{N}-\mathrm{C}>\mathrm{N}-\mathrm{T}>\mathrm{DM}-\mathrm{T}$. On contrast, the body weights of the DM-C achieved the maximum value at W3 and followed by a decrease trend at W4 and W5. At the end of experiment at W5, mice in the $\mathrm{N}-\mathrm{C}$ group had the greatest body weights while those in the DM-C had the least. Change in blood glucose of mice in the DM-C group and DM-T group are shown in Figure 1(B). The blood glucose of mice in the DM-C group elevated significantly at W2, fell slightly at W3 and then remained steady, while that in the DM-T group decreased continuously from W1 to W4 but slightly increased at W5. This suggests that continuing intragastric administration of COS could reduce the blood glucose level of diabetic mice and the difference in blood glucose level between COS administered group and control group increased as the feeding time increased.

\subsection{Analysis on Diversity of Mice Gut Microbiota Community}

ANOVA test indicated that in both the DM group and the normal blood glucose group there was no significant difference in $\alpha$-diversity (including species richness, Shannon index, phylogenetic diversity and evenness) $(P>0.05)$, suggesting that neither DM or intragastric administration of COS had a significant effect on the $\alpha$-diversity of gut microbiota in mice.

\subsection{Community Structure and Composition of Mice Gut Microbiota}

PCoA analysis based on Bray-Curtis dissimilarity (Figure 2) indicated significant difference in the community structure of gut microbiota in mice across the groups. The results of PERMANOVA analysis based on Bray-Curtis dissimilarity also showed that both the health status of mice $\left(\mathrm{R}_{\text {Health }}=0.536, P=0.001\right)$ and intragastric administration of COS $\left(\mathrm{R}_{\mathrm{Food}}=0.539, P=0.001\right)$ had a significant effect on the structure of gut microbiota in mice.

At the phylum level, among the dominant bacteria in gut microbiota of mice (with mean relative abundance $>1 \%$ in each group, Figure $3(\mathrm{~A})$ ), Bacteroidetes and Firmicutes had the highest abundance and were the absolute dominant microbiota in the gut of mice in the four groups. The ratio of Firmicutes/ Bacteroidetes in DM mice was significantly lower in the DM-T group than in the DM-C group. In mice of the normal blood glucose group, however, intragastric administration of COS made no difference. The total abundance of Actinobacteria had 
significant difference among the groups $(P<0.05)$, the relative abundance of Actinobacteria in gut of mice in the DM-T group was significantly higher than that in the DM-C group.
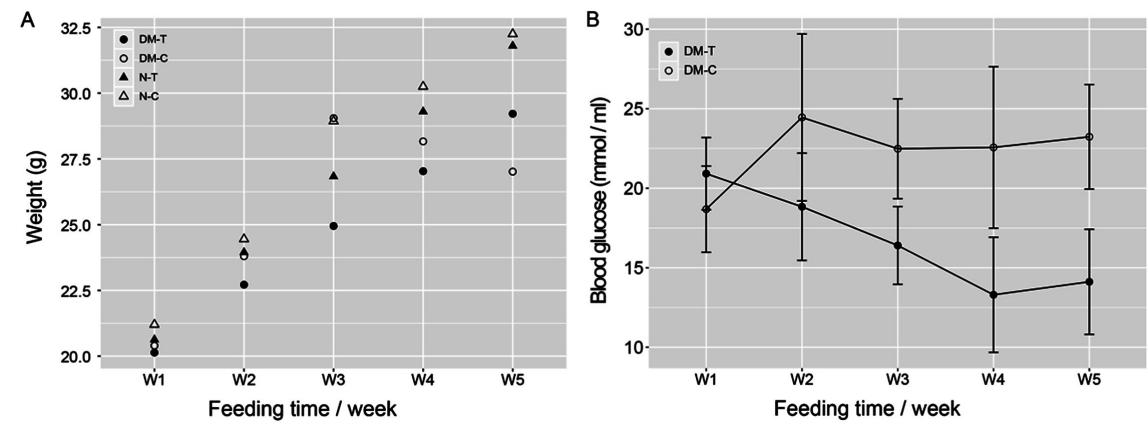

Figure 1. The profiles of weight (A) and blood glucose (B) of tested mice over time.

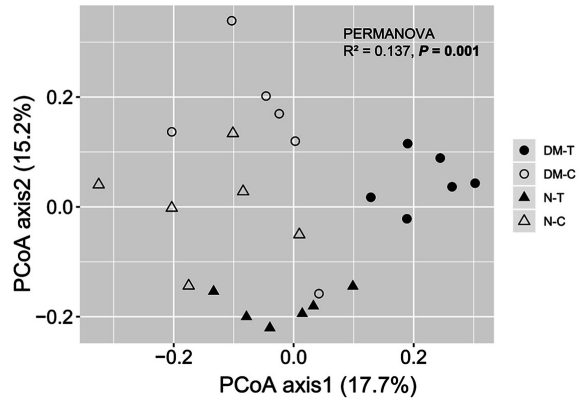

Figure 2. PCoA analysis of mice gut microbiota based on Bray-Curtis dissimilarity.

A

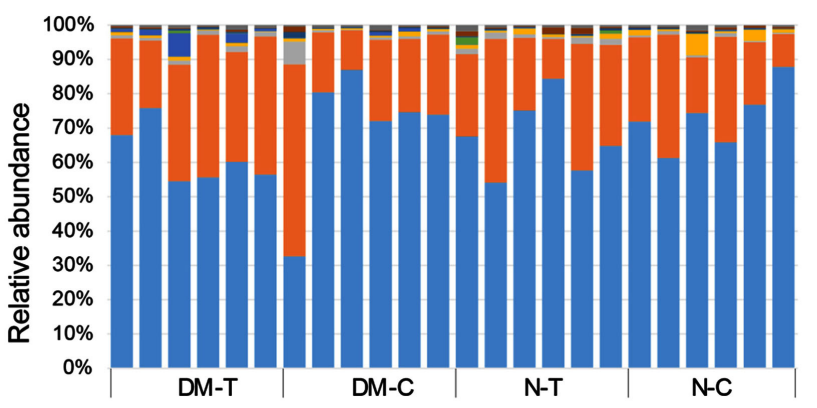

Others

- Deferribacteres

- Tenericutes

- Verrucomicrobia

Actinobacteria

- Betaproteobacteria

- Deltaproteobacteria

- Firmicutes

Bacteroidetes

B

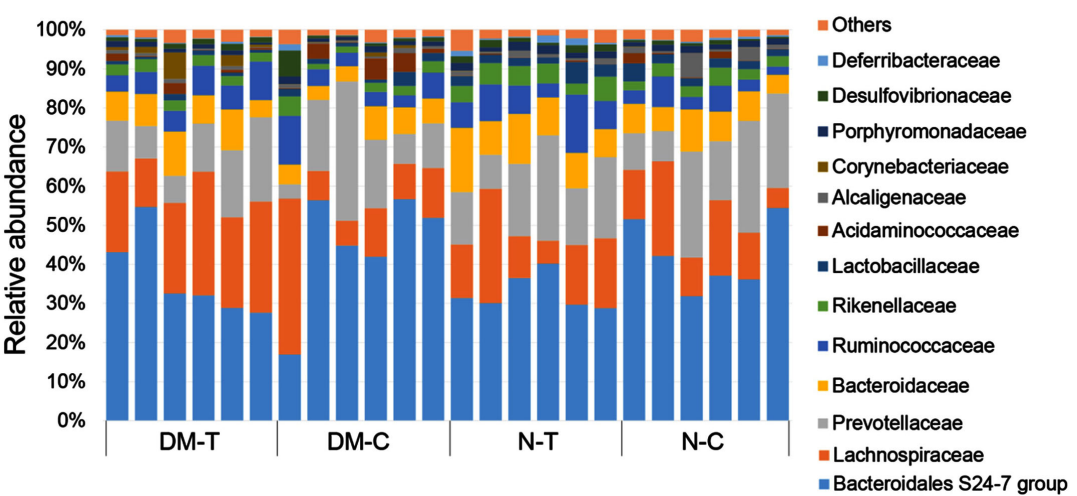

Figure 3. Composition of dominant bacteria (with mean relative abundance $>1 \%$ ) in gut microbiota of mice. (A) at phyla and proteobacterial classes level, (B) at family level. 
The composition of dominant bacteria in mice gut microbiota at family level is shown in Figure 3(B). The dominant families (with relative abundance $>1 \%$ ) mainly include four families, namely, Bacteroidales S24-7 group, Lachnospiraceae, Prevotellaceae and Bacteroidaceae. ANOVA test indicated that intragastric administration of COS influenced the abundance of Rikenellaceae and Bacteroidaceae in normal mice $(P<0.05)$, while the abundance of Corynebacteriaceae differed significantly between the DM-T group and DM-C group $(P<0.05)$. Corynebacteriaceae existed in gut of diabetic mice in great quantity, significantly greater in the DM-T group than in the DM-C group $(P<0.05)$. In the gut of mice in the normal groups, the relative abundance of Corynebacteriaceae was extremely low and intragastric administration of COS almost had no effect on it.

Table 1 presents the main OTUs of mice gut bacteria significantly influenced by intragastric administration of COS. Among the 14 OTUs of Bacteroidales S24-7 group, 5 had an abundance significantly decreasing along with the intragastric administration of COS in both the normal blood glucose groups and the DM groups, 8 decreasing significantly only in the DM-T group and 1 affected only in the N-T group. One OTU in family Lachnospiraceae was affected significantly only in the DM-T group and one was affected significantly only in the $\mathrm{N}-\mathrm{T}$ group.

Table 1. OTUs of mice gut microbiota with significant inter-group difference (One-way ANOVA).

\begin{tabular}{|c|c|c|c|c|c|}
\hline Most related OTU & Family & $\mathrm{F}$ & $P$ & $\mathrm{~N}-\mathrm{C} / \mathrm{T}$ & $\mathrm{DM}-\mathrm{C} / \mathrm{T}$ \\
\hline New.ReferenceOTU68 & Bacteroidales S24-7 group & 10.057 & 0.000 & Sig. & Sig. \\
\hline EF096612.1.1402 & Bacteroidales S24-7 group & 9.858 & 0.000 & Sig. & Sig. \\
\hline EF097238.1.1400 & Bacteroidales S24-7 group & 8.995 & 0.001 & Sig. & Sig. \\
\hline EU452486.1.1403 & Bacteroidales S24-7 group & 4.577 & 0.013 & Sig. & Sig. \\
\hline EF097214.1.1401 & Bacteroidales S24-7 group & 3.656 & 0.030 & Sig. & Sig. \\
\hline New.ReferenceOTU35 & Bacteroidales S24-7 group & 8.696 & 0.001 & No & Sig. \\
\hline HQ681769.1.1460 & Lachnospiraceae & 7.438 & 0.002 & No & Sig. \\
\hline EU512010.1.1420 & Bacteroidales S24-7 group & 7.182 & 0.002 & No & Sig. \\
\hline New.ReferenceOTU356 & Bacteroidales S24-7 group & 6.823 & 0.002 & No & Sig. \\
\hline New.CleanUp.ReferenceOTU19 & Bacteroidales S24-7 group & 6.690 & 0.003 & No & Sig. \\
\hline New.ReferenceOTU2401 & Bacteroidales S24-7 group & 6.048 & 0.004 & No & Sig. \\
\hline AB702728.1.1499 & Bacteroidales S24-7 group & 5.907 & 0.005 & No & Sig. \\
\hline New.ReferenceOTU37 & Bacteroidales S24-7 group & 5.756 & 0.005 & No & Sig. \\
\hline EF097757.1.1401 & Bacteroidales S24-7 group & 5.355 & 0.007 & No & Sig. \\
\hline GQ449002.1.1384 & Bacteroidaceae & 14.156 & 0.000 & Sig. & No \\
\hline New.ReferenceOTU34 & Rikenellaceae & 9.492 & 0.000 & Sig. & No \\
\hline GQ448253.1.1400 & Lactobacillaceae & 8.442 & 0.001 & Sig. & No \\
\hline New.ReferenceOTU300 & Bacteroidales S24-7 group & 6.489 & 0.003 & Sig. & No \\
\hline EU655974.1.1317 & Rikenellaceae & 3.937 & 0.023 & Sig. & No \\
\hline EU457759.1.1398 & Bacteroidaceae & 3.231 & 0.044 & Sig. & No \\
\hline
\end{tabular}




\section{Discussion and Conclusion}

With diabetic mice model as the object, this study investigated the effect of intragastric administration of COS on blood glucose of diabetic mice model and the gut microbiota of diabetic mice, using high-throughput sequencing technology. The level of blood glucose of mice in the COS intragastric administration groups was significantly lower than that of the control groups, demonstrating that intragastric administration of COS can continuously decrease the level of blood glucose. This is in agreement with the study of Wang M. R., et al. [11].

$\mathrm{DM}$ and intragastric administration of COS had little effect on the $\alpha$-diversity, but significant effect on the structure and composition of gut microbiota in mice, and the effect was more significant in the COS intragastric administration groups than in the normal blood glucose groups. Although at phylum level intragastric administration of COS had no significant effect on Bacteroidetes and Firmicutes in mice gut microbiota, inconsistent with the literature [7], at the OTU level, the abundance of OTUs of two phyla presented significant inverse change: 13 OTUs in Bacteroidales S24-7 group of phylum Bacteroidetes had a significant decrease in abundance while 1 OTU in phylum Firmicutes had a significant increase in abundance. It has been reported that the decrease of abundance of Bacteroidales S24-7 group was accompanied by improvement of symptoms of diabetes mellitus [21]. Moreover, the abundance of Actinobacteria was significant higher in the DM groups than in the normal groups. Intragastric administration of COS could significantly improve the abundance of phylum Actinobacteria and family Corynebacteriaceae under the phylum in the gut microbiota of diabetic mice, but had no significant effect on that in the gut microbiota of healthy mice. These results suggest that COS might improve the symptoms of diabetic mice via modulating the abundance of related bacteria in gut of mice and consequently alleviate diabetes symptoms.

\section{Acknowledgements}

This study is supported by Zhejiang Science and Technology Programme (2107C37105).

\section{Conflicts of Interest}

The authors declare no conflicts of interest regarding the publication of this paper.

\section{References}

[1] Kostic, A.D., Xavier, R.J. and Gevers, D. (2014) The Microbiome in Inflammatory Bowel Diseases: Current Status and the Future Ahead. Gastroenterology, 146, 1489-1499. https://doi.org/10.1053/j.gastro.2014.02.009

[2] Wang, T., Cai, G., Qiu, Y., et al. (2012) Structural Segregation of Gut Microbiota between Colorectal Cancer Patients and Healthy Volunteers. The ISME Journal, 6, 320-329. https://doi.org/10.1038/ismej.2011.109

[3] Fei, N. and Zhao, L. (2013) An Opportunistic Pathogen Isolated from the Gut of an 
Obese Human Causes Obesity in Germfree Mice. The ISME Journal, 7, 880-884. https://doi.org/10.1038/ismej.2012.153

[4] Arthur, J.C., Perez-Chanona, E., Muhlbauer, M., et al. (2012) Gut Inflammation Targets Cancer-Inducing Activity of the Microbiota. Science, 338, 120-123. https://doi.org/10.1126/science.1224820

[5] Backhed, F., Ley, R.E., Sonnenburg, J.L., et al. (2005) Host-Bacterial Mutualism in the Human Intestine. Science, 307, 1915-1920. https://doi.org/10.1126/science.1104816

[6] Eckburg, P.B., Bik, E.M., Bernstein, C.N., et al. (2005) Diversity of the Human Gut Microbial Flora. Science, 308, 1635-1538. https://doi.org/10.1126/science.1110591

[7] Larsen, N., Vogensen, F.K., Berg, F.W., et al. (2010) Gut Microbiota in Human Adults with Type 2 Diabetes Differs from Non-Diabetic Adults. PLoS ONE, 5, e9085. https://doi.org/10.1371/journal.pone.0009085

[8] Wang, Z.H., Jiang, Y.Y., Yu, X.H., et al. (2016) The Effect of Chitin and Its Water Soluble Derivatives on Immunity Function of Mice. Chinese Food Science, 37, 198-202.

[9] Oey, I., Verlinde, P., Hendnickx, M., et al. (2006) Temperature and Pressure Stability of L-Ascorbic Acid and/or [6s]-5-Methyltetrahydrofolic Acid: A Kinetic Study. European Food Research and Technology, 223, 71-77. https://doi.org/10.1007/s00217-005-0123-x

[10] Ni, T.Q. and Hu, S.Y. (2012) Progress of Research on the Pharmacological Effect and Clinical Application of Chitin and Its Derivative Chitosan. Drugs and Clinic, 27, 313-316.

[11] Wang, M.R., Yao, P. and Zhang, B. (2014) The Effect of Chitooligosaccharides on the Blood Glucose and Gut Microbiota of Mice with Diabetes Caused by Streptozocin-STZ. Chinese Journal of Biochemical Pharmaceutics, 34, 72-74.

[12] Terada, A., Hara, H., Sato, D., et al. (1995) Effect of Dietary Chitosan on Faecal Microbiota and Faecal Metabolites of Humans. Microbial Ecology in Health and Disease, 8, 15-21. https://doi.org/10.3109/08910609509141377

[13] Mrazek, J., Koppova, I., Kopecny, J., et al. (2010) PCR-DGGE-Based Study of Fecal Microbial Stability during the Long-Term Chitosan Supplementation of Humans. Folia Microbiologica, 55, 352-358. https://doi.org/10.1007/s12223-010-0057-y

[14] Azuma, K., Izumi, R., Kawata, M., et al. (2015) Effects of Oral Administration of Chitin Nanofiber on Plasma Metabolites and Gut Microorganisms. International Journal of Molecular Sciences, 16, 21931-21949. https://doi.org/10.3390/ijms160921931

[15] Chen, J.G., Mei, S., Fu, Y., et al. (2004) Study on Normal Reference Value of Fasting Blood Glucose in ICR Mice. Journal of Health Toxicology, No. 1, 61. (In Chinese)

[16] Ren, L., Li, B.L., Gao, S.Y., et al. (2001) Effects of Chitooligosaccharides on Blood Glucose and Intestinal Flora in Diabetic Mice. Chinese Journal of Biochemical Pharmaceutics, 22, 227-229. (In Chinese) https://doi.org/10.1016/S0378-5173(01)00879-1

[17] Parada, A.E., Needham, D.M. and Fuhrman, J.A. (2016) Every Base Matters: Assessing Small Subunit rRNA Primers for Marine Microbiomes with Mock Communities, Time Series and Global Field Samples. Environmental Microbiology, 18, 1403-1414. https://doi.org/10.1111/1462-2920.13023

[18] Caporaso, J.G., Kuczynski, J., Stombaugh, et al. (2010) QIIME Allows Analysis of High-Throughput Community Sequencing Data. Nature Methods, 7, 335-336. 
https://doi.org/10.1038/nmeth.f.303

[19] Magoč, T. and Salzberg, S.L. (2011) FLASH: Fast Length Adjustment of Short Reads to Improve Genome Assemblies. Bioinformatics, 27, 2957-2963.

https://doi.org/10.1093/bioinformatics/btr507

[20] Price, M.N., Dehal, P.S. and Arkin, A.P. (2010) FastTree 2-Approximately Maximum-Likelihood Trees for Large Alignments. PLoS ONE, 5, e9490.

https://doi.org/10.1371/journal.pone.0009490

[21] Hu, T., Wen, P., Shen, W., Liu, F., Li, Q., Li, E., Liao, S., Wu, H. and Zou, Y. (2019) Effect of 1-Deoxynojirimycin Isolated from Mulberry Leaves on Glucose Metabolism and Gut Microbiota in a Streptozotocin-Induced Diabetic Mouse Model. Journal of Natural Products, 82, 2189-2200.

https://doi.org/10.1021/acs.jnatprod.9b00205 